\title{
Indirekte Regelmechanismen der Stoffwechselgröße von Fischen
}

\author{
Peter W. Ruff und Ursula Zippel \\ Pbysiologisches Institut der Humboldt-Universität, Berlin
}

\begin{abstract}
Indirect regulatory mechanisms of metabolic rate in fishes. Metabolic rate does not strictly follow changes of influencing parameters. $\mathrm{O}_{2}$-consumption by fish is ordinarily said to be independent from $\mathrm{O}_{2}$-pressure throughout a wide range. This constancy is probably the result of a regulation. The metabolic rate itself or an auxiliary mechanism "before" or "after" metabolism may be regulated. On the basis of van DAM's paper (1938) and our own experiments, the problem whether or not $\mathrm{O}_{2}$-pressure in blood serves as a regulated auxiliary mechanism in order to maintain a certain metabolic constancy is discussed. The disadvantage of such a regulation would be the energy demand of increased ventilation and circulation as compensating mechanisms. In accordance with this assumption we have found a rise in $\mathrm{O}_{2}$ consumption at a somewhat reduced $\mathrm{O}_{2}$-pressure. Locomotory behaviour supports the constancy of metabolic rate; thus a contrary shift of the temperature preferendum after application of substances which influence metabolism has been found.
\end{abstract}

\section{EINLEITUNG}

Die Energiefreisetzung im Organismus ist letzten Endes an oxydative Vorgänge gebunden. Als Maß der Größe des oxydativen Stoff wechsels und damit des Energiewechsels dient seit alters her der $\mathrm{O}_{2}$-Verbrauch. Unter Annahme eines mittleren kalorischen O2-Aquivalents, das bei Aufnahme der gewöhnlichen arteigenen Nahrung annähernd gleich bleibt, ist dieses Maß hinreichend dem kalorischen Umsatz proportional.

Primär wird die Größe des oxydativen Stoffwechsels abhängen vom Angebot der Reaktionspartner, den Nährstoffen und dem Sauerstoff in der stoffwechselnden Zelle, von der Temperatur, vom Aktivitätszustand der Zelle und von hormonalen und pharmakologischen Einflüssen. Bei einer qualitativen Veränderung dieser Parameter wird in den meisten Fällen eine verhältnismäßig geringe Veränderung der Stoffwechselgröße beobachtet. Es sei an folgende Beispiele erinnert: Die Temperaturabhängigkeit von Stoffwechselreaktionsketten liegt oft niedriger als der van't Hoffschen Regel für chemische Prozesse entspricht (Precht 1955). Stroganov (1939) und Schlieper (1950) beschrieben eine Zone relativer Thermostabilität des Stoffwechsels, die sich über einen Temperaturbereich von $10^{\circ} \mathrm{C}$ erstrecken kann. Eine adaptive Rückführung der Stoffwechselgröße auf annähernd das Ausgangsniveau innerhalb von zwei Tagen nach Temperaturänderung wurde von Ріснотка (1958) am Beispiel des Kartoffelgewebes gezeigt. Nach Nahrungsaufnahme ist zwar eine Stoffwechselsteigerung im Sinne der 
spezifisch-dynamischen Wirkung zu beobachten, doch wird die Umsatzgröße nicht generell durch die aktuelle Nahrungszufuhr bestimmt. Die von SEguin \& Lavoisier (1789) angenommene Unabhängigkeit des Verbrauches des "air vital" von seinem Gehalt in der zugeführten Luft ließ sich zwar nicht absolut, aber doch weitgehend aufrechterhalten. Eine Dämpfung der Faktorenwirksamkeit auf den Umsatz ist im Rahmen der allgemeinen Homoiostase für den Homoiothermen naheliegend, obwohl die Stoffwechselgröße keine Zustandsgröße ist. So entscheidet die Wärmeproduktion nicht allein über die Körpertemperatur, sondern kann weitgehend durch veränderte Wärmeabgabe kompensiert werden. Im Interesse des Organismus, etwa als Leistungszuwachs, erfolgen Umsatzsteigerungen dagegen ohne weiteres. Ebenso erfolgt eine Adaptation der Stoffwechselgröße an langfristig veränderte Außenbedingungen, zum Beispiel verringerte Nahrungszufuhr, wobei die spezifisch-dynamische Wirkung hier abermals im Dienste der Erhaltung der Körpermasse auf Kosten der Stoffwechselkonstanz steht (LANG 1952).

Es erhebt sich die Frage, ob die beobachtete relative Konstanz das Ergebnis eines Regelvorganges ist oder ob eine vorgeschaltete Schrittmacherfunktion - wie im Falle der geringen Temperaturabhängigkeit der Diffusion - limitierend wirkt. Solche unregulierten Mechanismen mögen zwar beteiligt sein, aber die Gesamtheit der beobachteten Erscheinungen wird schwerlich darin ihre Erklärung finden. Regelvorgänge können die Stoffwechselgröße unmittelbar betreffen, diese selbst kann die geregelte Größe sein. Sie können aber auch „vor" oder "nach" dem Stoffwechsel liegen. Die Ansicht PFLÜGers (1872), daß die Zelle selbst ihren $O_{2}$-Verbrauch bestimme und daß darin „das wesentliche Geheimnis für die Regulation der durch den Gesamtorganismus verbrauchten Sauerstoffmenge " liege, entspricht der erstgenannten Möglichkeit. Eine Regulation "hinter" dem Stoffwechsel stellt die von Pichotra (1954) nachgewiesene Bedeutung der Thermoregulation für die $\mathrm{O}_{2}$-Aufnahme Homoiothermer dar. Hier ist eine Stoffwechselfolge, die Körpertemperatur, die geregelte Größe und stellt bei peristasebedingter primärer Anderung der O2-Aufnahme (durch verringerten $\mathrm{O}_{2}$-Druck) die Stoffwechselgröße auf das exforderliche Niveau der Wärmeproduktion ein. Die Feststellung Kroghs (1916), daß nicht die Oz-Spannung im Außenmedium, sondern im perizellulären Milieu entscheidend für den Verbrauch sei, legt die Bedeutung des arteriellen pog als Hilfsregelgröße (DRISCHEL 1952/53) des Stoffwechsels nahe. Die Feststellung eines der genannten Mechanismen schließt nicht die grundsätzliche Realisierung der anderen Möglichkeiten aus, sei es bei anderen Tieren, sei es nebeneinanderbestehend bei der gleichen Spezies. Allerdings ließe eine vollkommene Regelung einer Stoffwechselprämisse (po2) eine nachgeschaltete Regulation nicht in Gang kommen, doch ist eine Konstanterhaltung einer solchen Regelgröße in einem weiten Bereich sicherlich nicht gegeben.

\section{DIE O2-SPANNUNG IM BLUT ALS MUTMASSLICHE HILFSREGELGROSSE ZUR KONSTANTERHALTUNG DER STOFFWECHSELGROSSE}

Oberhalb eines kritischen Wertes des $\mathrm{O}_{2}$-Druckes wird im allgemeinen keine oder nur geringe Abhängigkeit des $\mathrm{O}_{2}$-Verbrauches von Fischen angenommen. Die in diesem 
Zusammenhang häufig herangezogenen Ergebnisse von HENze (1910) erscheinen bei näherer Betrachtung wenig beweiskräftig, doch konnten sogar gegenteilige Beobachtungen wie die von GaARder (1918) als Stütze einer Konstanzannahme herangezogen werden, wenn man daraus schließt, daß der Fisch sich ständig in Hypoxie befinde, der kritische Wert also oberhalb 160 Torr $\mathrm{pO}_{2}$ im Außenmedium liege. Ein Anstieg des $\mathrm{O}_{2}$-Verbrauchs mit fallendem $\mathrm{O}_{2}$-Druck bis herab zu einem (in diesem Falle wesentlich niedrigeren) kritischen Wert wurde zwar wiederholt bei Homoiothermen, aber bei Fischen wohl nur von van DAM (1938) beschrieben. Er beobachtete beim Aal (Anguilla valgaris L.) eine Steigerung der O2-Aufnahme um 21 bis $78 \%$ mit dem Maximum bei (nach unserer Berechnung) 40 Torr pog, an einer Regenbogenforelle (Salmo shasta JORDAN) bei 70 Torr p02 eine Steigerung der O2-Aufnahme um 66\%. In eigenen Versuchen (RuFf 1961) am Goldfisch (Carassius auratus L.) lag das Maximum bei höheren $\mathrm{O}_{2}$-Drucken (100 bis 110 Torr po2) und ergab in 20 Versuchen in diesem Bereich eine mittlere Steigerung der O2-Aufnahme um 39,3\%. Unsere Ergebnisse, die im offenen System (Durchfluß) bei titrimetrischer $\mathrm{O}_{2}$-Bestimmung gewonnen wurden, sind in der Tabelle 1 und als Säulen in Abbildung 1 dargestellt. Die Befunde von VAN DAM wurden zum Vergleich als senkrechte Linien eingetragen. VAN DAM führte die Umsatzsteigerung auf eine vermehrte Atemarbeit zurück. Wir schließen uns dieser Interpretation an, wobei wir aber die notwendigerweise gekoppelte Vergrößerung der Herzarbeit einbeziehen. Die Messung des Ventilationsvolumens durch VAN DAM ermöglicht unter seiner Annahme eine Berechnung der Umsatzsteigerung bezogen auf die Vermehrung des Ventilationsvolumens ( $\mathrm{ml} \mathrm{O} \mathrm{O}_{2}$-Mehrverbrauch/100 $\mathrm{ml}$ Wasser Mehrventilation). In Abbildung 2 sind diese Quotienten für Anguilla vulgaris links über den zugehörigen $\mathrm{O}_{2}$-Drucken und rechts über der prozentualen Ventilationszunahme aufgetragen. Leider läßt die geringe Anzahl von 6 Versuchen keine gute Korrelation erwarten. Es läßt sich im geprüften (niedrigen) Druckbereich keine Verschlechterung des Wirkungsgrades bei zunehmender Atemarbeit ablesen. Bei extrem niedrigem po2 (19 Torr unserer Berechnung) kann eine fünffache Ruheventilation bei $49 \%$ des Ruheumsatzes zustandekommen. Auch die Forelle bringt eine 3- bis 4fache Ausgangsventilation bei unverändertem oder verringertem $\mathrm{O}_{2}$-Verbrauch zustande. Beim Aal crhöht sich der $\mathrm{O}_{2}$-Verbrauch im Durchschnitt für 100\%ige Ventilationssteigerung um $8 \%$. Setzt man voraus, daß der Wirkungsgrad gleich bleibt und daß der $\mathrm{O}_{2}$-Mehrverbrauch nur der zusätzlichen Atemarbeit dient, so entfallen unter Normalbedingungen in Ruhe $8 \%$ des Umsatzes auf die Atemarbeit. Die bei 19 Torr pos beobachtete fünffache Ruheventilation würde demnach $81 \%$ der dort gemessenen $\mathrm{O}_{2}$-Aufnahme zu ihrer Deckung erfordern. Eine derartige Ventilationssteigerung im $\mathrm{O}_{2}$-Mangel wird nur kurzfristig unter Eingehen einer erheblichen Sauerstoffschuld möglich sein. In ähnlicher Weise war die gleiche Ventilationssteigerung um $297 \%$ bei der Regenbogenforelle einmal bei 73 Torr po2 verbunden mit einer Vergrößerung der $\mathrm{O}_{2}$-Aufnahme um $66 \%$ und ein anderes Mal bei 43 Torr poz verbunden mit einer Verringerung der $\mathrm{O}_{2}$-Aufnahme um $15 \%$. Man muß demnach zwischen diesen beiden $\mathrm{O}_{2}$-Druckwerten den kritischen Wert annehmen, bei dem nicht einmal der normale $\mathrm{O}_{2}$-Verbrauch aufrechterhalten werden kann.

Eine Mehrventilation bei gleichem Verbrauch würde zu einer geringeren Ausschöp- 
fung des Sauerstoffs in der Kiemenhöhle und dadurch zum Ansteigen des mittleren $\mathrm{O}_{2}$-Druckes führen. Dieser $\mathrm{O}_{2}$-Druck entspricht dem maximal durch Diffusion im arteriellen Blut erreichbaren. Die Kompensation eines initialen Absinkens des arteriellen pon ist nur durch eine echte Hyperventilation (über den Bedarf hinaus) möglich. Wenn, wie bei Spberoides, die Utilisation über einen gewissen poz-Bereich konstant bleibt (Nrcol 1960), wird bei Hyperpnoe zwar eine größere $\mathrm{O}_{2}$-Menge diffundieren,

Tabelle 1

Anderung des $\mathrm{O}_{2}$-Verbrauchs in Abhängigkeit vom $\mathrm{O}_{2}$-Druck

\begin{tabular}{|cccc|}
\hline $\begin{array}{c}\mathrm{O}_{2} \text {-Spannung } \\
\text { (Torr) }\end{array}$ & $\begin{array}{c}\text { Anzahl der } \\
\text { Versuche }\end{array}$ & $\begin{array}{c}\text { Mittlere Anderung } \\
\text { der Or-Aufnahme }(\%)\end{array}$ & $\begin{array}{c}\text { Streuung des } \\
\text { Mittelwertes }\end{array}$ \\
\hline $30-40$ & 4 & $-40,3$ & $\pm 7,4$ \\
$40-50$ & 4 & $\pm 28,1$ & $\pm 12,0$ \\
$50-60$ & 3 & $\pm 18,7$ & $\pm 1,6$ \\
$60-70$ & 5 & $+28,5$ & $\pm 11,2$ \\
$70-80$ & 10 & $+28,0$ & $\pm 9,1$ \\
$80-90$ & 8 & $+30,8$ &, \pm 3 \\
$90-100$ & 14 & $+36,0$ &, \pm 5 \\
$100-110$ & 20 & $+33,1$ & $\pm 10,0$ \\
$110-120$ & 12 & $+13,3$ & $\pm 16,1$ \\
$120-130$ & 6 & & $\pm 13,4$ \\
$130-140$ & 3 & & \\
\hline
\end{tabular}

aber der arterielle $\mathrm{O}_{2}$-Druck nicht ansteigen. Bei Tieren mit einem kontinuierlichen Ventilationsstrom kommt der $\mathrm{O}_{2}$-Druck an ihren respiratorischen Epithelien dem des umgebenden Milieus näher. Die geringe $\mathrm{O}_{2}$-Kapazität des Wassers macht eine solche Optimierung notwendig. Diese Tiere haben aber im Unterschied zu Lungenatmern keine großen Möglichkeiten, diesen p02 durch Hyperventilation zu erhöhen. Weitere Mechanismen, die den milieubedingten pog-Abfall dämpfen, wirken sich erst in den Gewebskapillaren aus: Verringerung der arterio-venösen $\mathrm{O}_{2}-$ Differenz und dadurch Steigerung des mittleren kapillaren poz durch Vergrößerung des Herzzeitvolumens und Verlagerung der Ausschöpfung in den steiler verlaufenden Teil der Oz-Dissoziationskurve des Hämoglobins. Die zuletzt genannte Teilkompensation ist ein Beispiel für einen "vor" dem Stoff wechsel liegenden, aber nicht geregelten Mechanismus.

Einen Schluß auf die Beteiligung "vor" oder "nach" dem Stoffwechsel liegender geregelter Größen dürfte der Zeitverlauf des $\mathrm{O}_{2}$-Verbrauchs nach rechteckiger Verstellung des $\mathrm{p}_{2}$ im Außenmilieu zulassen. Wenn die ursprüngliche geregelte Größe eine Stoff wechselfolge ist, wie bei den von PicHotKa (1954) untersuchten Homoiothermen, wird die initiale Auslenkung der zunächst noch nicht geregelten Umsatzgröße besonders auffällig sein. Die Dauer der Einstellbewegung hängt von der Organisationshöhe des untersuchten Tieres ab; beim Meerschweinchen (PrсHотkA 1955) nimmt sie Minuten in Anspruch. Bei Evertebraten, die vermutlich den zellulären Stoffwechsel direkt regulieren, dauert die Einstellung Stunden, wenn wir die von REHREN (1954/55) mitgeteilten Kurven im Unterschied zu der Autorin in diesem Sinne interpretieren dürfen. In unseren Versuchen an Carassizs auratus sahen wir keine Einstellvorgänge, was unseres Erachtens für die starke Beteiligung vorgeschalteter Regelvorgänge spricht, die einen 


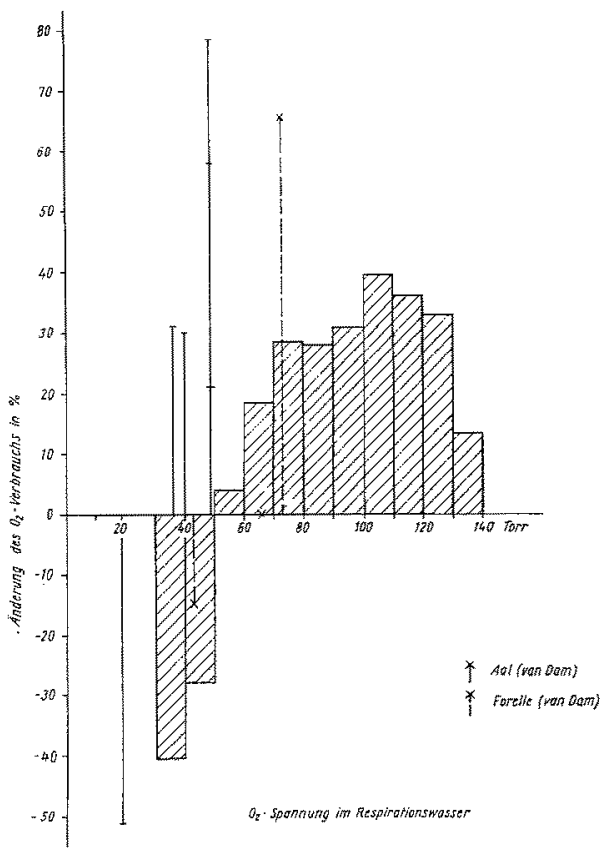

Abb. 1: Prozentuale Anderung des $\mathrm{O}_{2}$-Verbrauches in Abhängigkeit vom $\mathrm{O}_{2}$-Druck des Respirationswassers bei Carassius auratus (schraffierte Säulen). Zum Vergleich sind Werte für Anguilla vulgaris und Salmo shasta (senkrechte Linien; nach vAN DAM 1938) eingetragen

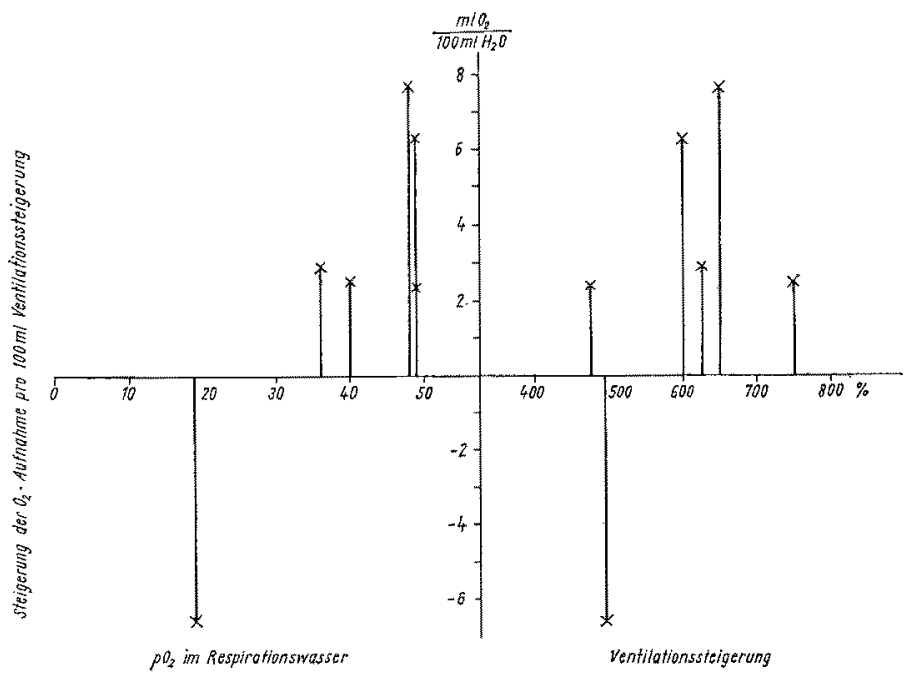

Abb. 2: Steigerung der $\mathrm{O}_{2}$-Aufnahme pro $100 \mathrm{ml}$ Ventilationssteigerung bei Anguilla vulgaris in Abhängigkeit vom $\mathrm{O}_{2}$-Druck im Respirationswasser (links) beziehungsweise von der prozentualen Ventilationssteigerung (rechts), berechnet nach Angaben von VAN DAM (1938) 
initialen Abfall des $\mathrm{O}_{2^{-}}$Verbrauchs gar nicht erst zustande kommen lassen beziehungsweise überkompensieren.

Die Kompensierung des $\mathrm{O}_{2}$-Mangels durch einen energiefordernden Prozeß, also unter Erhöhung des $\mathrm{O}_{2}$-Verbrauches, ist eine unvermeidliche Paradoxie, vor die sich bei der Kurzzeitadaptation auch die landlebenden Vertebraten gestellt sehen, ob nun dieser Leistungszuwachs im Bruttoumsatz zum Ausdruck kommt oder nicht. Wird bei niedrigen $\mathrm{O}_{\mathrm{g}}$-Drucken dieser Mehrverbrauch nicht mehr gedeckt, so wird nach Erschöpfung anoxydativ erschließbarer Energiequellen schnell der kritische Wert unterschritten. Die beobachtete Steigerung des $\mathrm{O}_{2}$-Verbrauchs bei mäßig erniedrigtem po macht eine Definitionserweiterung des Begriffes "kritischer Wert" dahingehend notwendig, daß es sich um den erniedrigten $\mathrm{O}_{2}$-Druck handelt, bei dem der im Luftsättigungsbereich beobachtete $\mathrm{O}_{2}$-Verbrauch wieder erreicht und unterschritten wird.

\section{DAS LOKOMOTORISCHE VERHALTEN IM DIENSTE DER KONSTANTHALTUNG DER STOFFWECHSELGROSSE}

Die Anderung des Außenmilieus durch Aufsuchen optimaler Umgebungsbedingungen ist in ähnlicher Weise energiefordernd wie die genannten viszeralen Kompensationsmechanismen. In den meisten Fällen wird die Kleinheit des Gradienten lediglich eine ungerichtete Lokomotionssteigerung erlauben. Sie ist risikobehaftet: der sicheren Umsatzsteigerung steht eine sehr ungewisse Verbesserung der Milieufaktoren gegenüber. Dieses Risiko wird oft nicht eingegangen; es liegen widersprüchliche Ergebnisse über die Veränderung der lokomotorischen Aktivität bei niedrigem po2 vor (FRY 1957, Nicol 1960). Wir konnten an Carassius auratus keine eindeutige Aktivitätsänderung im Sauerstoffmangel beobachten, vor allem keine Steigerung der äußeren Aktivität, die den erhöhten Sauerstoffverbrauch bedingen oder mitbedingen könnte. In gesonderten Versuchen erbrachte das Durchströmen der Versuchsgefäße mit Stickstoff auch innerhalb der gleichen Spezies unterschiedliche Reaktionen.

In einem ganz anderen Sinne konnten wir ein Ortswahlverhalten beim Guppy (Lebistes reticulatus Peters) beobachten, das pharmakologisch verursachte Anderungen der Stoffwechselgröße zumindest richtungsmäßig ausgleicht. Es gelang uns, durch Vorbehandlung mit Chininum hydrochloricum die Vorzugstemperatur um $1^{0} \mathrm{C} \mathrm{zu}$ erhöhen und durch Vorbehandlung mit Thyrotan (Glandulae thyreoideae siccatae, 8 Stoffwechseleinheiten/Liter) um $0,6^{\circ} \mathrm{C}$ zu senken. Die Unterschiede zu den unbehandelt gemessenen Vorzugstemperaturen sind mit einer Irrtumswahrscheinlichkeit $\mathrm{P}<$ $1 \%$ signifikant. Wir erklären die Befunde mit einer Veränderung der Rezeptorentemperatur auf Grund veränderter Wärmeproduktion. Dabei wird ein Temperaturbereich aufgesucht, in dem die Umsatzgröße entsprechend der Stoffwechselgeschwindigkeits-Temperaturrelation in entgegengesetzter Weise verändert wird wie durch das vorher verabfolgte Pharmakon. Der $\mathrm{O}_{2}$-Verbrauch konnte in der Temperaturorgel nicht bestimmt werden, so daß wir keine quantitative Aussage über die Ergiebigkeit dieser Gegenbewegung machen können. Beziehungen des Temperaturpräferendums zur Stoffwechselregulation bestehen sicher, wenn auch die Annahme, daß die Vorzugstem- 
peratur mit einem Stoffwechselminimum zusammenfalle (HERTer 1962), für Poikilotherme sich schwerlich generalisieren läßt und für Homoiotherme nicht gilt (PrснотKA 1961).

\section{ZUSAMMENFASSUNG}

1. Die relative Peristaseunabhängigkeit der Stoffwechselgröße wird an Hand von Literaturbeispielen aufgezeigt. Von der Mehrzahl der Autoren wird eine weitgehende Unabhängigkeit des $\mathrm{O}_{2}$-Verbrauches rom $\mathrm{O}_{2}$-Druck bei Fischen angenommen.

2. Es liegt nahe, eine solche Konstanz als Ergebnis eines Regelvorganges aufzufassen, wobei die geregelte Größe die Stoffwechselgröße selbst oder eine Hilfsregelgröße darstellen könnte, die entweder "vor" oder "hinter" dem Stoffwechsel liegt.

3. Die Möglichkeit, daß bei Fischen eine solche Hilfsregelgröße im $\mathrm{O}_{2}$-Druck des Blutes zu suchen sei, wird an Hand der Literatur (VAN DAM 1938) und eigener Untersuchungen diskutiert. Eine solche Regelung ist insofern nicht vollkommen, als bei erniedrigtem $\mathrm{poz}_{\mathrm{z}}$ die kompensierenden Mechanismen ihrerseits energiefordernd den Stoffwechsel belasten. Entsprechend wurde eine Steigerung der $\mathrm{O}_{2}$-Aufnahme bei mäßig erniedrigtem $\mathrm{p}_{2} 2$ beobachtet.

4. Es wird ferner das lokomotorische Verhalten im Dienste der Konstanterhaltung des Umsatzes besprochen und über eine Abschwächung der Wirkung stoffwechselwirksamer Pharmaka durch eine gegenläufige Verlagerung der Vorzugstemperatur berichtet.

\section{ZITIERTE LITERATUR}

DAM, L. VAN, 1938. On the utilization of oxygen and regulation of breathing in some aquatic animals. Groningen, Diss.

Drischel, H., 1952/53. Bausteine einer dynamischen Theorie der vegetativen Regulation. Wiss. Z. Univ. Greifswald (Math.-nat, R.) 2, 99-164.

FRY, F. E. J., 1957. The aquatic respiration of fish. In: The physiology of fishes. Ed. by M. E. Brown. Acad. pr., New York, 1, 1-63.

GaARDER, T., 1918. Ober den Einfluß des Sauerstoffdruckes auf den Stoffwechsel. Nach Versuchen an Karpfen. Biochem. Z. 89, 94-125.

HenzE, M., 1910. Uber den Einfluß des Sauerstoffdruckes auf den Gaswechsel einiger Meerestiere. Biochem. Z. 26, 255-278.

Herter, K, 1962. Der Temperatursinn der Tiere. Ziemsen, Wittenberg, 80 pp.

KROGH, A., 1916. The respiratory exchange of animals and man. Longmans, London.

LANG, K., 1952. Physiologie der Ernährung. In: Die Ernährung. Hrsg. von K. Lang und R. Schoen. Berlin, Springer.

Nicor, J. A. C., 1960. The biology of marine animals. Pitman \& Sons, London, 707 pp.

PFLÜGER, E., 1872. Über die Diffusion des Sauerstoffs, den Ort und die Gesetze der Oxydationsprozesse im thierischen Organismus. Pflügers Arch. ges, Physiol. 6, 43-64.

PrсноткA, J., 1954. Die Sauerstoffaufnahme als geregelte Größe. Klin. Wschr. 32, 1021.

- 1958. Uber das Wesen biologischer Reaktionen auf meteorologische Einflüsse. Med.-met. Hft. 13, 38-48.

- 1961. Körpertemperatur und Stoffwechselgröße. Arch. Tierernäbr. Beibeft 8, 16-35.

- Creutzfeldt, O. \& Höfler, W., 1955. Die Bewegungen der Körpertemperatur und der 
Sauerstoffaufnahme bei veränderter Wärmeabgabe im Sauerstoffmangel. Arch. exp. Path. Pharmak. 225, 335-341.

Precht, H., Christophersen, J. \& Hensel, H., 1955. Temperatur und Leben. Springer, Berlin, 514 pp.

ReHren, E., 1954/55. Die Abhängigkeit der Atmung vom Sauerstoffpartialdruck bei wirbellosen Wassertieren. Zool. Jb. (Allg. Zool. Physiol. Tiere) 65, 237-266.

Ruff, P., 1961. Die Oz-Aufnahme von Süßwasserfischen bei geringer Senkung der $\mathrm{O}_{2}$-Spannung. Pflügers Arch. ges. Physiol. 274, 73.

SchliEPER, C., 1950. Temperaturbezogene Regulationen des Grundumsatzes bei wechselwarmen Tieren. Biol. Zbl. 69, 216-226.

Segurn, A. \& Lavorsier, A. L., 1789. Premier mémoire sur la respiration des animaux. (Zit.) In: Pichotka, J.: Der Gesamtorganismus im Sauerstoffmangel. Handbuch der allgemeinen Pathologie. Springer, Berlin, 4 (2), 498-568 (1957).

Stroganov, N. S., 1939. The effect of temperature on the interrelations of gas exchanges in the perch Perca fuviatilis (russ.). Fiziol. Żh. SSSR 26a, 69-82. 